\title{
Basic MR sequence parameters systematically bias automated brain volume estimation
}

Sven Haller ${ }^{1,2,3,4}$, Pavel Falkovskiy, $5,6,7$, Reto Meuli ${ }^{6}$, Jean-Philippe Thiran ${ }^{7}$, Gunnar Krueger ${ }^{8}$, Karl-Olof Lovblad ${ }^{1,9}$, Alexis Roche ${ }^{5,6,7}$, Tobias Kober ${ }^{5,6,7}$, and Bénédicte Maréchal ${ }^{5,6,7}$

\begin{abstract}
${ }^{1}$ Faculty of Medicine of the University of Geneva, Geneva, Switzerland, ${ }^{2}$ Affidea Centre de Diagnostique Radiologique de Carouge CDRC, Geneva, Switzerland, ${ }^{3}$ Department of Surgical Sciences, Radiology, Uppsala University, Uppsala, Sweden, ${ }^{4}$ Department of Neuroradiology, University Hospital Freiburg, Freiburg, Germany, ${ }^{5}$ Advanced Clinical Imaging Technology (HC CMEA SUI DI BM PI), Siemens Healthcare AG, Lausanne, Switzerland, " ${ }^{6}$ Department of Radiology, University Hospital (CHUV), Lausanne, Switzerland, 'LTS5, École Polytechnique Fédérale de Lausanne, Lausanne, Switzerland, ${ }^{8}$ Siemens Medical Solutions USA, Inc., Boston, ME, United States, ${ }^{9}$ University Hospitals of Geneva, Geneva, Switzerland
\end{abstract}

\section{Synopsis}

Standard MR parameters, notably spatial resolution, contrast and image filtering, systematically bias results of automated brain MRI morphometry by up to $4.8 \%$. This is in the same range as early disease-related brain volume alterations, for example in Alzheimer's disease. Automated brain segmentation software packages should therefore require strict MR parameter selection or include compensatory algorithms to avoid MR-parameter-related bias of brain morphometry results.

\section{Introduction}

Automated brain MRI morphometry, including hippocampal volumetry for Alzheimer's disease, is increasingly recognized as a biomarker. Consequently, a rapidly increasing number of software tools have become available. We tested whether modifications of MR protocol parameters which are often adapted in clinical routine systematically bias automated brain MRI segmentation results.

\section{Material and Methods}

This study was approved by the local ethical committee and included 21 consecutive patients (13 females, mean age $75.8 \pm 13.8$ years) undergoing clinical brain MRI for workup of cognitive decline. MR imaging was performed on a whole-body 1.5T clinical MR scanner (MAGNETOM Aera, Siemens Healthcare Ltd., Erlangen, Germany) using a 20-channel head coil. Three different 3D T1-weighted sagittal volumes (see Figure 1) were obtained using the magnetization-prepared rapid gradient-echo (MPRAGE) pulse sequences ${ }^{1,2}$ employing the imaging parameter settings described in Figure 2. 
Brain segmentation was performed by two different analysis tools, the MorphoBox prototype ${ }^{3,4}$ and Freesurfer ${ }^{5}$ using standard parameters. Estimated volumes of the following brain tissues and structures were analyzed with the R software package (version 3.1.1): total intracranial volume (TIV), grey matter (GM), cortical grey matter (cGM), white matter (WM), hippocampus, ventricles, and cerebellum.

In order to assess the presence of a potential systematic bias in the volumetric results, relative volume differences (RVD) between the reference protocol $\left(\mathrm{V}_{\mathrm{r}}\right)$ and each variant $\left(\mathrm{V}_{\mathrm{V}}\right)$ were computed for each structure as:

$$
\left.\operatorname{RVD}\left(\mathrm{V}_{\mathrm{r}}, \mathrm{V}_{\mathrm{v}}\right)=100 \mathrm{~V}_{\mathrm{v}}-\mathrm{V}_{\mathrm{r} /(}\left(\mathrm{V}_{\mathrm{v}}+\mathrm{V}_{\mathrm{r}}\right) / 2\right),
$$

where $\mathrm{RVD}\left(\mathrm{V}_{\mathrm{r}}, \mathrm{V}_{\mathrm{v}}\right)$ is in the range [-200\%,200\%].

ADNI-2 and LOCAL- protocols were used as the reference protocol $\left(\mathrm{V}_{\mathrm{r}}\right)$ in all subsequent analyses. Relative volume differences were averaged across the subjects. The statistical significance of the difference from the zero median in relative volumetric differences was tested using the Wilcoxon signed-rank test as the differences were not expected to be normally distributed. Bonferroni correction was used to correct for multiple comparisons.

It has been shown previously that the variance of volume differences does not significantly change across different systems (different field strength, different vendors), but systematic offsets in volumes may be present ${ }^{6}$. Therefore, to compare our results to previously reported scan-rescan reproducibility studies ${ }^{7,8,9,10}$, absolute relative volume differences (ARVD) between the reference protocol $\left(\mathrm{V}_{\mathrm{r}}\right)$ and each variant $\left(\mathrm{V}_{\mathrm{v}}\right)$ were recomputed for each structure as:

$$
\operatorname{ARVD}\left(\mathrm{V}_{\mathrm{r}}, \mathrm{V}_{\mathrm{v}}\right)=100\left|\mathrm{~V}_{\mathrm{v}}-\mathrm{V}_{\mathrm{r}}\right| /\left(\left|\mathrm{V}_{\mathrm{v}}+\mathrm{V}_{\mathrm{r}}\right| 2\right)
$$

where $\mathrm{ARVD}\left(\mathrm{V}_{\mathrm{r}}, \mathrm{V}_{\mathrm{v}}\right)$ is in the range $[0 \%, 200 \%]$.

ADNI-2 and LOCAL- protocols were used as the reference protocol $\left(\mathrm{V}_{\mathrm{r}}\right)$ in all subsequent analysis. Absolute relative volume differences were averaged across the subjects.

\section{Results}

\section{Effect of spatial resolution - 1.2 versus $1.0 \mathrm{~mm}$}

The comparison of ADNI2 versus LOCAL- protocols with the FreeSurfer segmentation tool revealed significant changes in TIV, GM, cGM, ventricles, and hippocampus volumes (see Figure 4A), even though they were hard to identify upon visual inspection (see Figure 3 ). The respective median RVDs were 2.42\% ( $p<0.01), 3.14 \%(p<0.001), 4.52 \%(p<0.001), 2.40 \% \quad(p<0.001)$, and $-3.81 \%$ $(p<0.05)$. For hippocampus volumes, the median ARVD was $4.23 \pm 5.97 \%$. Segmentation results obtained with the MorphoBox segmentation tool revealed significant changes in TIV and WM volumes (see Figure 4B); the respective median RVDs were $1.78 \%(p<0.001)$ and $1.60 \%(p<0.01)$. For hippocampus volumes, the median ARVD was $4.55 \pm 4.68 \%$. Note that the relative volume differences correspond to fixed offsets in segmentation results and do not represent scan-rescan variability of each protocol.

\section{Effect of image filtering}

The comparison of LOCAL- versus LOCAL+ protocols with the FreeSurfer segmentation software revealed a significant change in WM and ventricle volumes. The respective RVDs for WM and ventricle volumes were: $-1.82 \% \quad(p<0.05)$, and $-0.98 \% \quad(p<0.05)$. The, median ARVD of the hippocampus volumes was $2.99 \pm 7.70 \%$ (see Figure 5A). Using the MorphoBox segmentation tool, significant changes in TIV and WM volumes were observed. The respective RVDs of TIV and WM volumes were: $-0.28 \%(p<0.05)$, and $-4.84 \%(p<0.05)$. The median ARVD of the hippocampus volumes was $3.39 \pm 3.91 \%$ (see Figure $5 \mathrm{~B}$ ). 


\section{Discussion}

A simple change of MR parameters, notably spatial resolution, contrast and image filtering, may systematically bias results of automated brain MRI morphometry by up to $4.8 \%$. This is in the same range as early disease-related brain volume alterations, for example in Alzheimer's Disease ${ }^{11}$. Automated brain segmentation software packages should therefore require strict MR parameter selection or include compensatory algorithms ${ }^{12}$ to avoid MR-parameter-related bias of brain morphometry results.

\section{Acknowledgements}

No acknowledgement found.

\section{References}

1. Blaimer M, Breuer FA, Mueller $M$ et al. 2D-GRAPPA-operator for faster 3D parallel MRI. Magn Reson Med 2006; 56(6):-1359-1364

2. http://adni.Ioni.usc.edu/methods/documents/mri-protocols/

3. Roche A, Ribes D, Bach-Cuadra $M$ et al. On the convergence of EM-like algorithms for image segmentation using Markov random fields. Med Image Anal 2011; 15(6):830-839

4. Schmitter D, Roche A, Maréchal B et al. An evaluation of volume-based morphometry for prediction of mild cognitive impairment and Alzheimer's disease. Neuroimage Clinical 2015; 77-17

5. Fischl B. FreeSurfer. Neuroimage 2012; 62(2):-774-781

6. Jovicich J, Czanner S et al. MRI-derived measurements of human subcortical, ventricular and intracranial brain volumes: Reliability effects of scan sessions, acquisition sequences, data analyses, scanner upgrade, scanner vendors and field strengths. Neuroimage 2009; 46(1):177-192

7. Jovicich $\mathrm{J}$, Marizzoni $\mathrm{M}$ et al. Brain morphometry reproducibility in multi-center $3 \mathrm{~T}$ MRI studies: A comparison of cross-sectional and longitudinal segmentations. Neuroimage 2013; 83:472-484

8. Kruggel F, Turner J. et al. Impact of scanner hardware and imaging protocol on image quality and compartment volume precision in the ADNI cohort. Neuroimage 2010; 49(3):21232133

9. Morey R.A, Selgrade E et al. Scan-rescan reliability of subcortical brain volumes derived from automated segmentation. Human Brain Mapping 2010; 31(11):1751-1762

10. Reuter $\mathbf{M}$, Schmansky $\mathbf{N}$ et al. Within-subject template estimation for unbiased longitudinal image analysis. Neurolmage 2012; 61(4):1402-1418

11. Frankó E, Joly O, for the Alzheimer's Disease Neuroimaging Initiative (2013) Evaluating Alzheimer's Disease Progression Using Rate of Regional Hippocampal Atrophy. PLoS ONE 8(8): e71354

12. Falkovskiy $\mathbf{P}$, Brenner D, Feiweier $T$, Kannengiesser $S$, Maréchal B, Kober $T$, Roche A, Thostenson K, Meuli R, Reyes D, Stoecker T, Bernstein MA, Thiran JP, Krueger G. Comparison of accelerated T1-weighted whole-brain structural-imaging protocols. Neuroimage. 2015 Aug 20;124(Pt A):157-167 
Figures

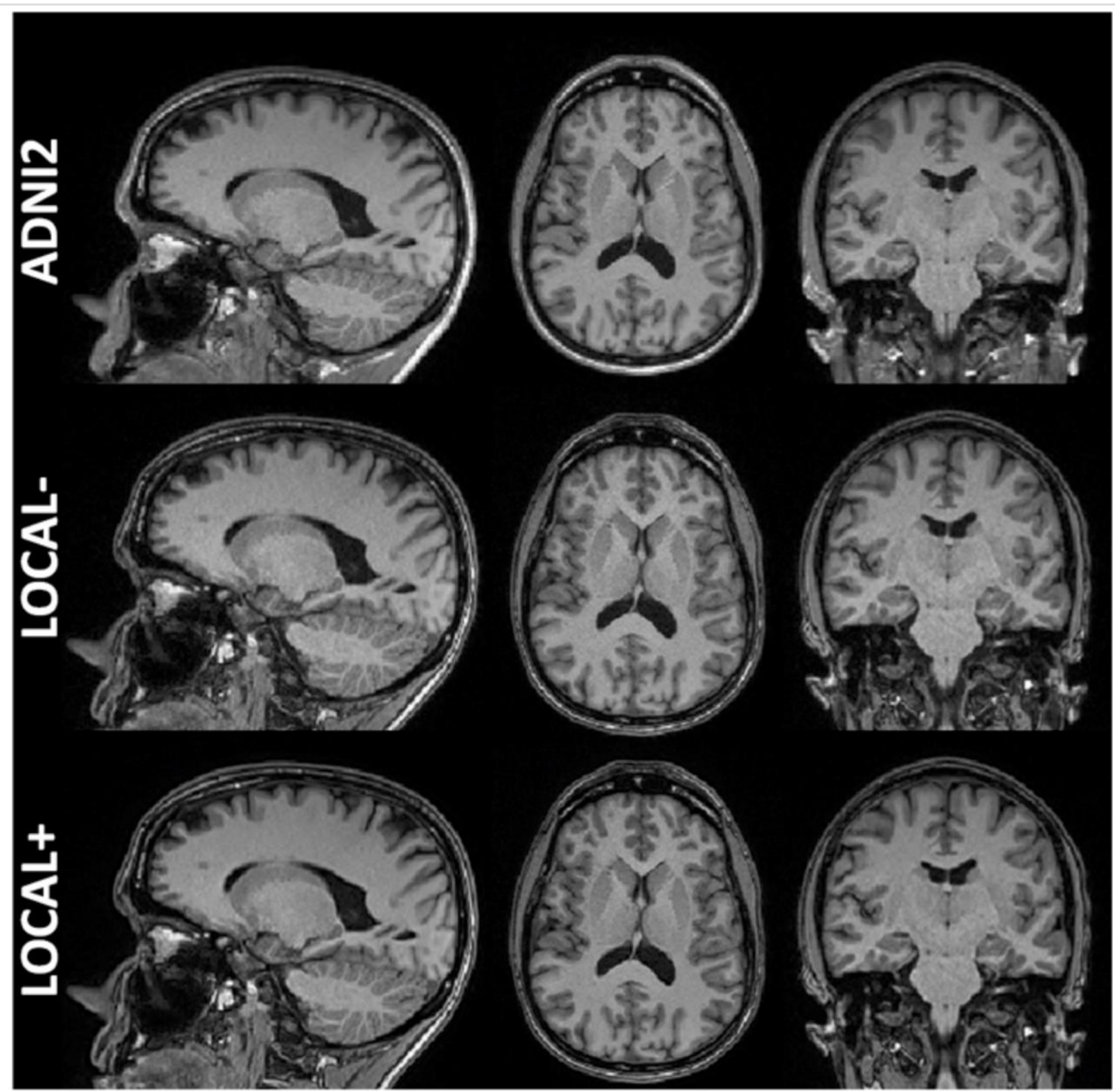

Figure 1. Example of the three different parameter settings for the MPRAGE sequences in coronal, sagittal, and axial slices 


\begin{tabular}{rccc} 
& ADNI2 & LOCAL- & LOCAL+ \\
\hline Voxel size $\left(\mathrm{mm}^{3}\right)$ & $1.25 \times 1.25 \times 1.2$ & $0.97 \times 0.97 \times 1$ & $0.97 \times 0.97 \times 1$ \\
$\mathrm{TR}(\mathrm{ms})$ & 2400 & 2200 & 2200 \\
$\mathrm{TI}(\mathrm{ms})$ & 1000 & 900 & 900 \\
Bandwidth $(\mathrm{hz} / \mathrm{px})$ & 180 & 150 & 150 \\
FOV read/phase $(\mathrm{mm})$ & $240 / 240$ & $250 / 240$ & $250 / 240$ \\
Edge enhancement filter & OFF & OFF & ON \\
\hline
\end{tabular}

Figure 2. Overview of the essential parameters of the three different MPRAGE protocols.

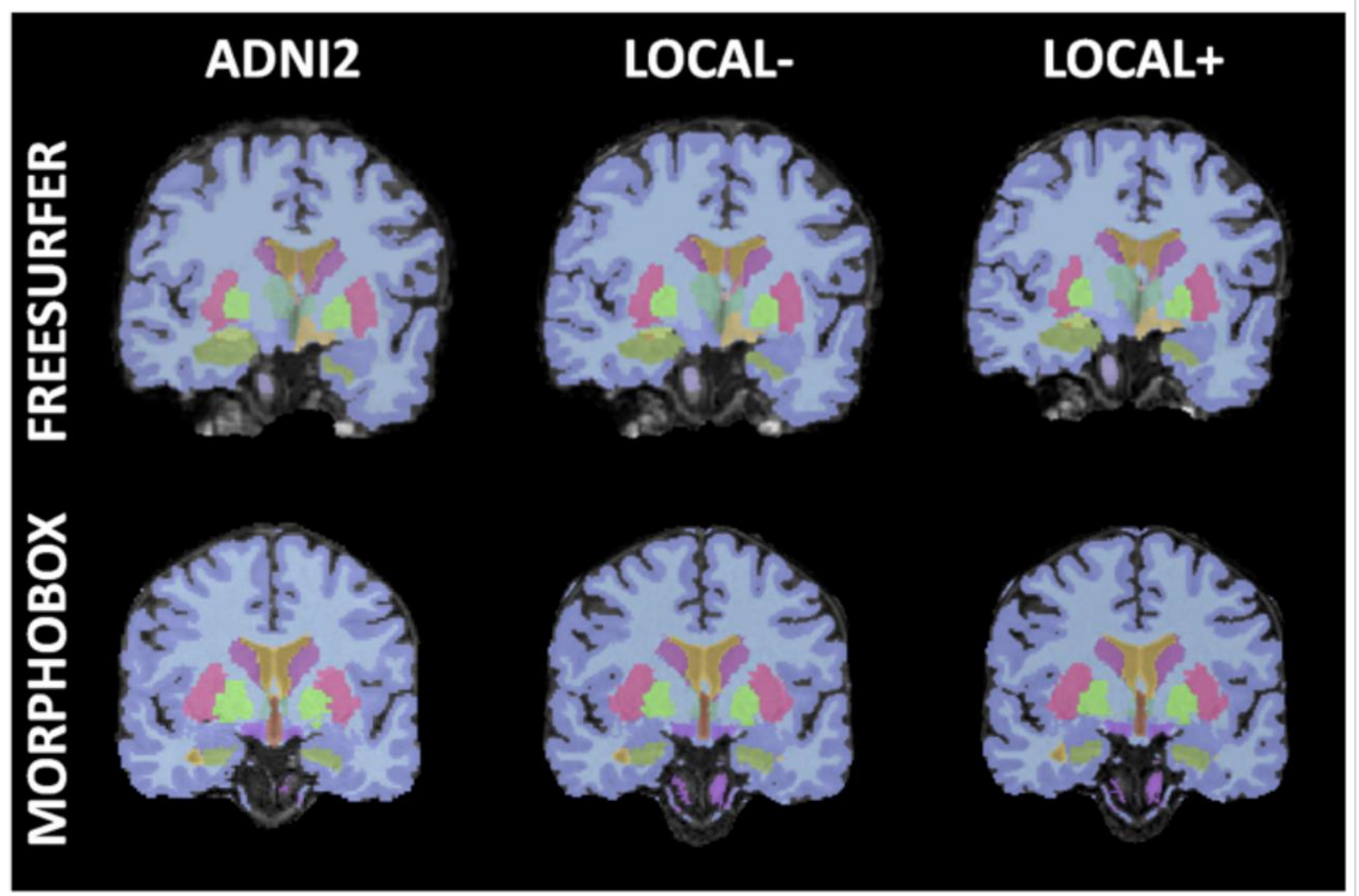

Figure 3. Example of coronal TIV extraction and segmentation obtained with Freesurfer and Morphobox for the three MPRAGE parameter settings 


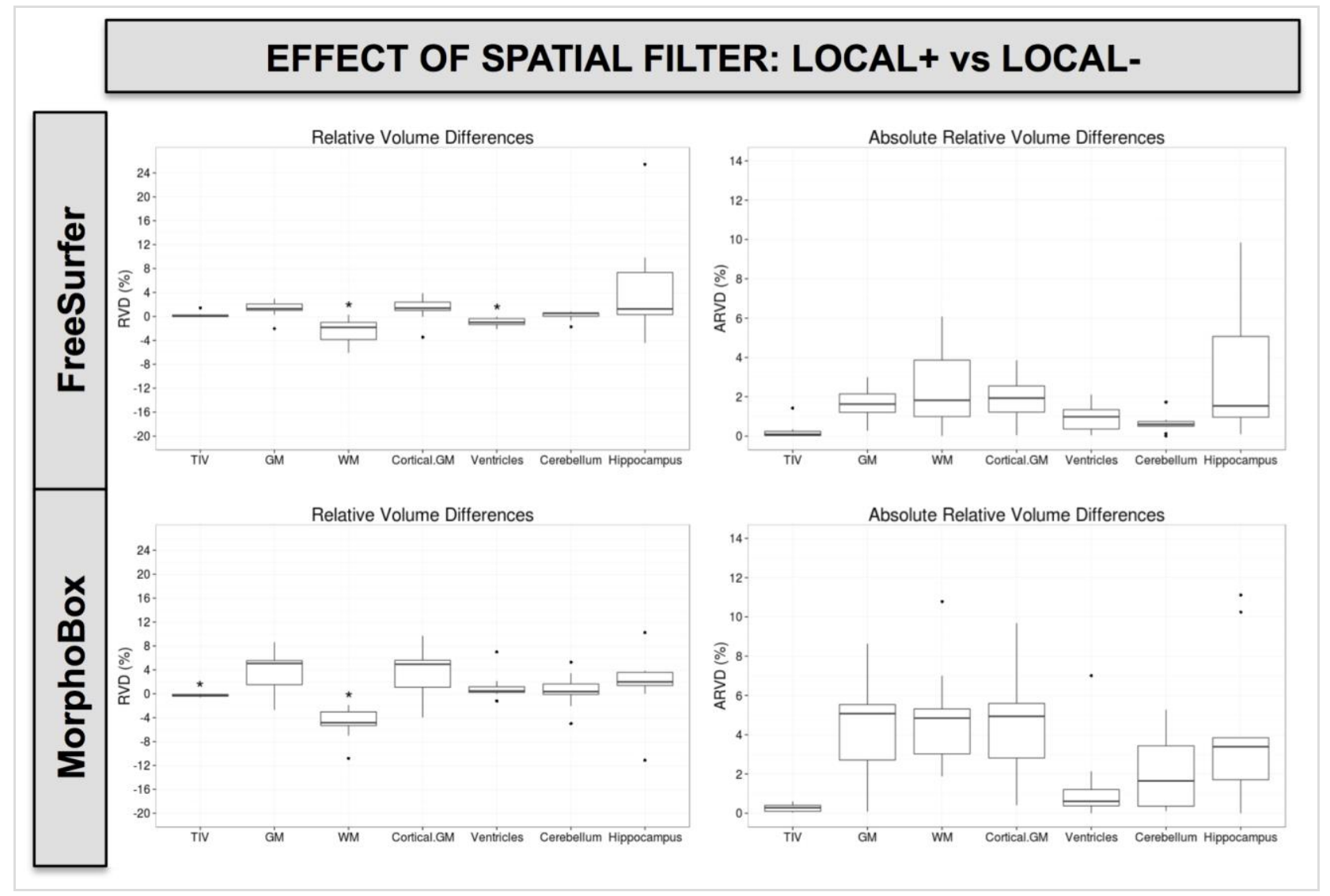

Figure 5. Variability of the brain segmentation results for the effect of spatial resolution and contrast by the comparison of ADNI2 versus LOCAL-. Note that the RVDs correspond to fixed offsets in segmentation results and do not represent scan-rescan variability of each protocol. TIV: total intracranial volume, GM: total grey matter, WM white matter, Cortical GM: only cortical grey matter. * $\mathrm{p}<0.05,{ }^{* *} \mathrm{p}<0.01,{ }^{* *} \mathrm{p}<0.001$ - corrected for multiple comparisons 


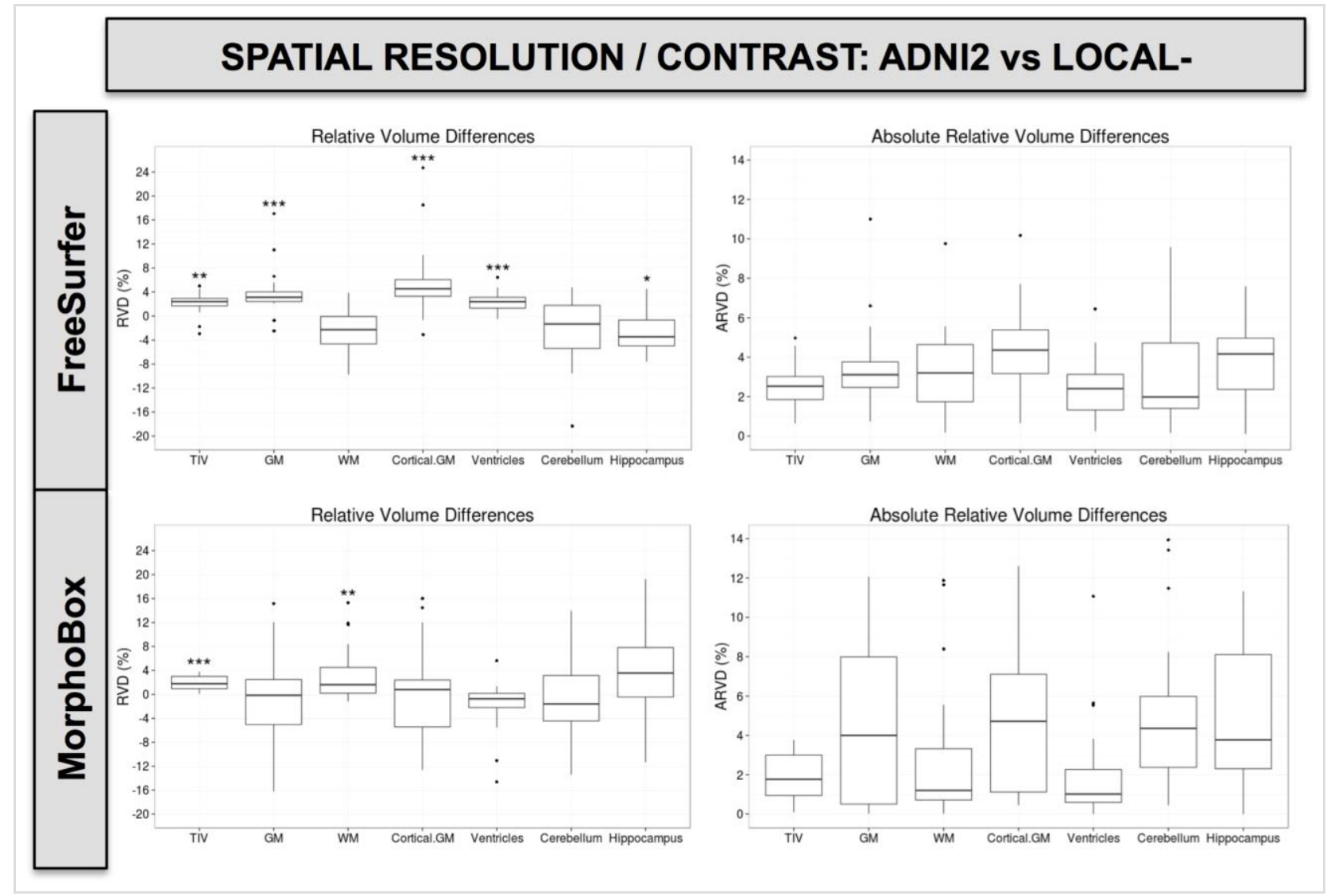

Figure 4. Variability of the brain segmentation results for the effect of image filtering (present versus absent filter) by the comparison of LOCAL- versus LOCAL+. Abbreviations equivalent to Figure 5

Abstract ID: 676 